\section{Fractional laser and laser assisted corticosteroid delivery for hypertrophic scars in thermal burns}

\author{
Ni Putu Susari Widianingsih, ${ }^{1}$ \\ Cita Rosita S. Prakoeswa ${ }^{2}$ \\ ${ }^{1}$ Surabaya Skin Centre Clinic, Jl. Prof. \\ Dr. Moestopo 175 Surabaya; \\ ${ }^{2}$ Dermatology and Venereology \\ Department, Faculty of Medicine, \\ Universitas Airlangga - Dr Soetomo \\ General Hospital, Surabaya, Indonesia
}

\begin{abstract}
The unprecedented survival of individuals who sustain acute burns and other trauma has increased the necessity for effective modalities in the treatment and rehabilitation of patients. Hypertrophic scars and contractures are common following thermal burns injury. Cutaneous scars can be complex and thus the approach to therapy is often multimodal with only moderate success. Intralesional corticosteroids have long been a main treatment of hypertrophic and restrictive scars. Recent advance in laser technology and applications now provide additional options for improvements in function, symptoms, and cosmesis. The ablative zones created by fractional ablative lasers may also be used in the immediate post-operative period to enhance delivery of drugs and other substances. This case report shows the successful use of fractional laser assisted corticosteroid to treat burn scars. It is need more extensive clinical trial to prove this method can be a therapeutic option for the extensive cutaneous scarring in burn patients.
\end{abstract}

\section{Introduction}

Scars from following any insult to the deep dermis as a result of the complex physiologic wound healing cascade which can be temporally grouped into three distinct phases (inflammation, proliferation, and remodeling). ${ }^{1}$ Immediately following wounding, platelet degranulation and activation of the complement and clotting cascades from a fibrin clot for hemostasis, which acts as a scaffold for wound repair. Platelet degranulation is responsible for the release and activation of an array of potent cytokines and growth factors, which serve as chemotactic agents for the recruitment of macrophages, neutrophils, fibroblast, and others. Recruited fibroblasts synthesize a scaffold of extracellular matrix (ECM) which builds a structural framework to bridge the wound and allow vascular ingrowth. Myofibroblasts help initiating wound contraction. Once the wound is closed, the immature scar transition into the final maturation phase, where abundant ECM is degraded and immature type III collagen is modified into mature type I collagen. ${ }^{1}$

Treating burns poses a great clinical challenge in that the scars formed following thermal or chemical injury are some of the worst scars seen in clinical practice. If a patient survives a burn injury, there can be both physical and psychologically devastating effects. ${ }^{2}$

Multiple studies on hypertrophic scar or keloid formation have led to a multitude of therapeutic strategies to prevent or improve keloid and hypertrophic scar formation and have been reviewed in a plethora of articles, such as intralesional cryotherapy, intralesional 5-fluorouracil, interferon, and bleomycin. ${ }^{1}$ Recent advances in laser technology and applications now provide additional options for improvements in function, symptoms, and cosmesis. ${ }^{3}$ Fractional photothermolysis has successfully treated a wide variety of dermatologic conditions including rhytids, pigmented lesions, melisma, acne scars, surgical scars, and actinic keratosis. $^{2}$ Ablative fractional laser (AFL) treatment facilitates delivery of topical drug deeply into the skin by creating vertical channels. ${ }^{4}$ This lasers create zones of ablation at variable depths of the skin with the subsequent induction of a wound healing and collagen remodeling response. ${ }^{3}$

\section{Case Report}

A 22-year-old woman who had been burned in a grease fire about 1 year before she came to the clinic, looked for a treatment for her scars. Treatment for the burns included debridement without skin grafting. After the wound cured, she only got topical cream to reduce the scar. She had a light scar contractures of the right hand inter-digital spaces and restricted movement of the wrist in daily activity. She also had keloidal scarring of her right and left wrists and elbows with pigment irregularities. Her face was revealed rough texture and mild erythema, without any hypertrophic scar.

Treatment of the facial scars and also her both arms were used a combination of fractional $\mathrm{CO} 2$ laser and topical triamcinolone acetonide suspension at a concentration of $10 \mathrm{mg} / \mathrm{ml}$ immediately after the laser procedure. Before the laser procedures, we gave the skin conditioning to the patient using tretinoin $0.05 \%$ every night and sun-
Correspondence: Ni Putu Susari Widianingsih, Surabaya Skin Centre Clinic, J1. Prof. Dr. Moestopo 175 Surabaya, Indonesia. Tel.: +6231.5999595.

E-mail: susy_vq@yahoo.com, sbyskincentre@yahoo.co.id.

Key words: thermal burns, hypertrophic scars, fractional laser, corticosteroid, laser assisted corticosteroid delivery.

Contributions: The authors contributed equally.

Conflict of interest: the authors declare no potential conflict of interest.

Received for publication: 1 February 2019. Accepted for publication: 20 February 2019.

This work is licensed under a Creative Commons Attribution-NonCommercial 4.0 International License (CC BY-NC 4.0).

(C) Copyright N.P.S. Widianingsih and

C.R.S. Prakoeswa, 2019

Licensee PAGEPress, Italy

Dermatology Reports 2019; 11(s1):8073

screen for the day. The laser procedure repeated for 5 times with 4-6 weeks interval for her arms, and only once treatment for her face. Fractional $\mathrm{CO}_{2}$ laser setting parameter were 25 watt in power, dwell time $800 \mu \mathrm{s}$, spacing time $800 \mu \mathrm{m}$, with 2 and 3 stacks for the keloidal scaring for just one pass (fluence ranged from 3.02 to 4.54 $\mathrm{J} / \mathrm{cm} 2$ at a treatment density $7.3 \%$ ). Treatment parameter utilized a lower parameter setting (fluence $1.51 \mathrm{~J} / \mathrm{cm} 2$ at asame treatment density). Immediately after the fractional $\mathrm{CO} 2$ laser procedure, a thin layer of triamcinolone acetonid suspension was drizzled over the site and rubbed gently over the ablated columns. After treatment care, patient was instructed to apply an antibiotics ointment (mupirocin 2\%) twice a day until healed. Patients were also directed to apply a physical sunscreen and avoid sun exposure. Following once treatments, the appearance of the skin on her face had significantly improved, with smoother skin surface. And after 5 treatments session of her arms, also gave a good improvement. Of the four improvement categories measured, the texture improvements was very good ( $>75 \%$ improvement), hypertrophy became thinner and dyschromia gave a good results $(50-75 \%$ improvement) after 3 months of the last laser procedures. The overall improvement reached a good improvement (50-75\% improvement). Patient also reported increased range of motion in wrist doing activities of daily life. The patients experienced moderate erythema and edema immediately post-treatment; 
the hyperpigmentation post inflammatory reaction was subsides soon after 4 weeks of the treatment. Treatments were well-tolerated and no adverse effects were reported (Figures 1 and 2).

\section{Discussion}

Scarring from thermal injuries occurs from a variety of sources including electrical burn, thermal burns from scalding water, grease, and direct burn from fires. Unfortunately, these burns frequently involve the face and hands where the can have significant morbidity. ${ }^{2}$ Treatment of often required a multimodal approach to therapy. Intralesional corticosteroids have been a mainstay in the treatment of hypertrophic scars for decades, leading to improvements through a variety of mechanisms including diminished collagen synthesis and increased collagen degradation. Furthermore, injection of triamcinolone acetonide is often painful and consistent scars, and also need a skilled injector because the precise placement of the drug severe cutaneous scars can be complex and dosing is difficult to achieve throughout the

can avoid adverse sequelae such as fat atrophy. In contrast, topical application of triamcinolone acetonide after fractional resurfacing is painless and may be applied with greater uniformity. ${ }^{3}$ The advent of fractional laser technology within the last decade has significantly increased potential treatment options for patients with disfiguring and disabling scars. ${ }^{3}$ The process of fractional resurfacing stimulates collagen formation in the dermis and causes remodeling at this level. Whereas fractional laser resurfacing has largely been used to resurface skin for aesthetic indications including photodamage, its use for the treatment of atrophic and surgical scars suggests that it may be highly useful in other types of scarring. There is a great deal of experience with fractional resurfacing of the skin and the histological data documents the type and the depth of injuries caused when fractional resurfacing lasers are used to treat atrophic scars. ${ }^{2}$

Ablative fractional laser-assisted corticosteroid delivery may take advantage of the newly formed channels to penetrate uniformly and deeply into dermal scars. Current ablative fractional laser devises have a significant benefit being tunable and thus creating channels of a predetermined
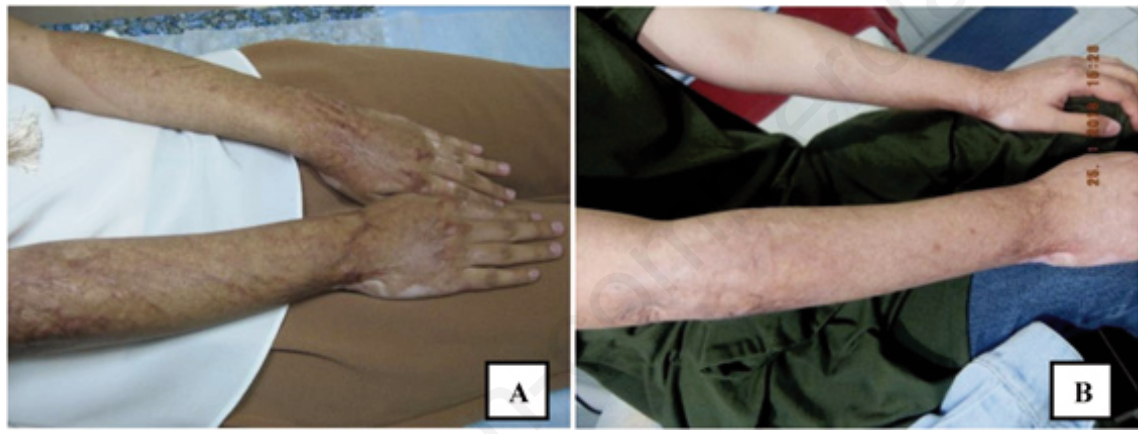

Figure 1. Hand scar and depigmentation following thermal burns (A), and after 5 times laser assisted delivery systems treatment using triamcinolone acetonide solution (B).

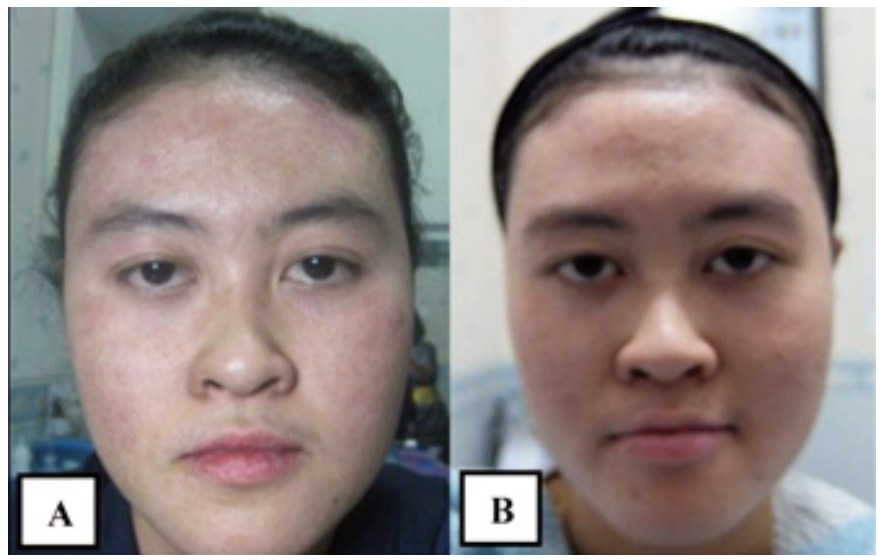

Figure 2. Facial skin before (A) and after (B) one treatment procedure (fractional laser and laser assisted corticosteroid delivery). depth and density. Previous histologic studies in normal skin have documented complete re-epithelialization within 48 hours after ablative fractional $\mathrm{CO}_{2}$ laser treatment. The preference of the authors is to apply the triamcinolone within 2 minutes of fractional treatment at the earliest phases of the inflammatory cascade. Capillary action likely facilitates the passage of the triamcinolone acetonide suspension through the channels without the need for an occlusive dressing. The termed of this mode called as laser assisted delivery systems, or LADS. ${ }^{3-6}$

\section{Conclusions}

Combination of fractional $\mathrm{CO}_{2}$ laser and laser assisted corticosteroid delivery for hypertrophic scar in thermal burns in this case offers a significant improvement, safe and effective. Side effects were limited and all transient. Further investigation is required to determine if same-session combination fractional laser and topical corticosteroid therapy is more effective compared to either modality alone, as well as to determine other variables such as optional laser settings and drug dosing.

\section{References}

1. Gauglitz GG. Management of keloids and hypertrophic scars: current and emerging options. Clinical, Cosmetic and Investigational Dermatology 2013:6:103-114.

2. Waibel J, Beer K. Case Reports: Fractional Laser Resurfacing for Thermal Burns. Journal of Drugs in Dermatology, 2008:7: 59-61

3. Waibei JS, Wulkan AJ, Shumaker PR. Treatment of Hypertrophic Scars Using Laser and Laser Assisted Corticosteroid Delivery. Lasers in Surgery and Medicine, 2013:45:135-140

4. Cavalie M, Sillard L, Montaudie H, Bahadoran P, Lacour JP, Passeron T. Treatment of keloids with laser-assisted topical steroid delivery: a retrospective study of 23 cases. Dermatologic Therapy, 2014:1-5.

5. Oni G, Lequeux C, Cho M, Zhang D, Lazcarno E, Brown S, Kenkel J. The Use of A Fractional Ablative Laser to Deliver Adipocyte Derived Stem Cells Transdermally - A Feasibility Pilot Study. Aesthet Surg J. 2013 Jan;33(1):109-16 PMID:23277622.

6. Waibel Jr, Beer Kenneth. Ablative Fractional Laser Resurfacing for the treatment of a third-degree burn.Journal of drugs in dermatology 2009:09. 